Research Article

\title{
Can blood groups, neutrophil-lymphocyte ratio and mean platelet volume predict mortality in critically ill patients developing acute kidney injury?
}

\begin{abstract}
Introduction and Aim: Acute kidney injury (AKI) in the recent years, is one of the main causes of morbidity and mortality in critically ill patients. Neutrophil lymphocyte ratio (NLR) is a novel and practical indicator of inflammation, and its effectiveness has been demonstrated in many diseases.

Material and Method: We retrospectively reviewed the medical records of totally 300 patients diagnosed with AKI, admitted to our intensive care unit between January 2010 and December 2017. As laboratory parameters, blood urea nitrogen (BUN) and creatinine levels, and glomerular filtration rate (GFR) as calculated according to MDRD formula were assessed both on the first day (BUN1, Cre1, GFR1) and last day (BUN2, Cre2, GFR2) of admission. C-reactive protein (CRP) level, white blood cell (WBC) count, neutrophil, lymphocyte and platelet values, mean platelet volume (MPV) and neutrophil lymphocyte ratio (NLR) were recorded.
\end{abstract}

Results: There was no association of blood groups with mortality or with other laboratory parameters (respectively $\mathrm{p}=0.986, \mathrm{p}=0.456, \mathrm{p}=0.872$ all values not significance $\mathrm{p}>0,05$ ) There was no statistically significant relationship of MPV value with mortality $(\mathrm{p}=0,314)$. Statistically, NLR showed very strong association with both mortality and AKI development (respectively $\mathrm{p}<0.0001, \mathrm{p}<0.0001$ ). There was also very strong association between mortality and other laboratory parameters (BUN1, BUN2, GFR1, GFR2, WBC, Platelet, NLR; all $\mathrm{p}<0.0001$ ).

Conclusion: MPV and blood groups are not predictors of mortality in critically ill patients. The change in NLR can be an important follow-up parameter to predict survival of critically ill patients. As NLR increases, so does the rate of intensive care mortality and AKI risk.

Keywords: acute kidney injury, neutrophil-lymphocyte ratio, mean platelet volume, blood group, mortality

\section{Introduction and aim}

Acute renal failure (ARF) is described as deterioration of renal functions where elimination of nitrogen waste products (urea) is restrained and body's fluid-electrolyte balance is impaired as a result of sudden decrease in glomerular filtration rate within hours to days. Since there is no complete biochemical definition of ARF, more than 35 definitions have been proposed so far. ARF is a conventional term that is frequently used for patients admitted to intensive care unit due to acute dialysis requirement. However, evidence shows that even a minimal decline in renal function has profound effect on mortality and morbidity. ${ }^{1,2}$ For this reason, the term ARF has been replaced by the term "acute kidney injury" (AKI) that was proposed by Acute Kidney Injury Network. ${ }^{3}$ Despite all the advanced life-support systems and treatment options, AKI is still an important cause of mortality and morbidity for patients monitored in intensive care units (ICU). Epidemiological data regarding AKI varies greatly in the literature. While its prevalence varies between $1-31 \%$, associated mortality rates are between $28-82 \%{ }^{4,5}$ The reason for this wide range is the difference between the criteria used to diagnose AKI. The most frequent cause of AKI in patients admitted to ICU is sepsis and septic shock. AKI occurs in various degrees and is generally progressive, and it is often described as a syndrome due to these properties. Although it is potentially reversible, AKI is an important cause of chronic renal failure. AKI frequently accompanies other severe diseases, and causes significant increases in mortality and morbidity rates. The prevalence of AKI among patients monitored in ICU is $1-25 \%$, and $5 \%$ of these patients require dialysis. ${ }^{6-8}$
Volume 8 Issue I - 2017

\author{
Ebru Canakci,' Ahmet Karatas, ${ }^{2}$ Yasemin \\ Kaya $^{3}$ \\ 'Ordu University, School of Medicine, Department of Anesthesia \\ and Reanimation, Ordu, Turkey \\ ${ }^{2}$ Ordu University, School of Medicine, Department of Internal \\ Medicine, Nephrology Branch, Ordu, Turkey \\ ${ }^{3}$ Ordu University, School of Medicine, Department of Internal \\ Medicine, Ordu, Turkey
}

Correspondence: Ebru Canakci, Ordu University School of Medicine Department of Anesthesia and Reanimation, Turkey, Email canakciebru@gmail.com

Received: May 08, 2017| Published: May 17, 2017

Systemic inflammatory response is associated with changes in white blood cell count in the peripheral blood, especially neutrophilia that is seen concurrent with relative lymphocytopenia. ${ }^{9}$ Neutrophil lymphocyte ratio (NLR) is a practical, repeatable and inexpensive marker that can be used in assessment of systemic inflammation. ${ }^{10}$ Mean platelet volume (MPV) is an indicator of platelet function and activation. ${ }^{11}$ While children and young adults have higher MPV values, MPV does not vary between the sexes. ${ }^{12}$ Increased MPV is an indicator of megakaryocytic enlargement occurring as a response to thrombopoietic stress. Large platelets can be defined as stressplatelets. MPV is increased when there is increased destruction of peripheral platelets, and is decreased when there is impairment of platelet production. ${ }^{13}$ Since larger platelets are more reactive, MPV is regarded as an indicator of increased cardiovascular disease risk in the general population. Several studies have shown MPV as an indicator for atherosclerotic diseases..$^{14}$ In addition to atherosclerosis, platelet volume is also increased in the presence of atherosclerotic risk factors including hypertension, hyperlipidemia, diabetes mellitus, end-stage renal disease and obesity. ${ }^{14-16}$

Blood groups were discovered in the $19^{\text {th }}$ century, and they are kind of cellular identity that is determined by the antigenic structure present on the surface of red blood cells. The blood group system discovered by Landsteiner is the general blood group system that is known as the ABO blood groups. Another commonly used blood grouping system is known as the Rh system, and depends on the presence or absence of this antigenic structure. $\mathrm{ABO}$ and $\mathrm{Rh}$ blood group system is currently most commonly used grouping system used 
today. Nevertheless, many blood group systems have been developed in addition to the $\mathrm{ABO}$ and $\mathrm{Rh}$ system, and many studies have been conducted on this subject. Numerous studies from different fields have examined the importance of blood group systems in tissue and organ transplantations and the genetic basis of blood groups. ${ }^{17}$ Regarding the diseases associated with mortality, early detection and diagnosis have utmost significance in terms of treatment success. Diseases that are considered as causes of mortality in especially the developed countries include cancers and cardiovascular diseases, which are thought to have a genetic background. The treatment of these diseases is quite cumbersome and expensive. Despite all the troubles and long treatment processes, it is not possible to completely cure these diseases. Nonetheless, as stated before, early diagnosis is very important regarding the course and treatment of these diseases. Considering all these reasons, it is important to investigate the relationship between some common diseases and the blood groups as they are very easily detectable genetical properties that are free from the influence of environmental factors. ${ }^{17,18}$

In this retrospective study, we aimed to investigate the relationship between mortality and blood groups, neutrophil lymphocyte ratio (NLR) and mean platelet volume (MPV) in critically ill patients developed AKI, additionally to determine whether NLR and MPV have predictive value regarding AKI development.

\section{Material and methods}

Following approval from Ordu University Clinical Studies Ethics Committee (Issue Nr:2016-14), medical records of 300 patients admitted to General Intensive Care Unit of Ordu University Training and Research Hospital and diagnosed with AKI were reviewed. Demographical properties (age, weight, height, sex) of all patients were recorded. Diagnosis at admission, accompanying comorbidities, whether mechanical ventilation was applied, whether the case was discharged or deceased, were noted for every case.As laboratory parameters, blood urea nitrogen (BUN) and creatinine levels, and glomerular filtration rate (GFR) as calculated according to MDRD formula were assessed both on the first day (BUN1, Cre1, GFR1) and last day (BUN2, Cre2, GFR2) of admission. C-Reactive Protein Table I Frequency table based on patients' demographic variables and examined parameters

\begin{tabular}{|c|c|c|c|}
\hline \multicolumn{2}{|l|}{ Variables } & Frequency & $\%$ \\
\hline \multirow{4}{*}{ ABO Groups } & 0 & 56 & 18.4 \\
\hline & $A$ & 187 & 61.5 \\
\hline & B & 36 & 11.8 \\
\hline & $A B$ & 21 & 6.9 \\
\hline \multirow{2}{*}{$\mathrm{RH}$} & Negative & 89 & 29.3 \\
\hline & Positive & 211 & 69.4 \\
\hline \multirow{2}{*}{ Sex } & Male & 144 & 48 \\
\hline & Female & 156 & 52 \\
\hline \multirow{2}{*}{ Renal failure } & $\mathrm{AKI}$ & 135 & 44.4 \\
\hline & Non-AKI & 165 & 54.3 \\
\hline \multirow{2}{*}{ Mechanical ventilation } & MV applied & 142 & 47.3 \\
\hline & MV not applied & 158 & 52.7 \\
\hline \multirow{2}{*}{ Discharge state } & Exitus & 146 & 48.7 \\
\hline & Discharged & 154 & 51.3 \\
\hline \multirow{11}{*}{ Diagnosis } & CVA & 42 & 13.8 \\
\hline & Pneumonia & 36 & 11.8 \\
\hline & Intoxication & 26 & 8.6 \\
\hline & $\mathrm{CHF}$ & 36 & 11.8 \\
\hline & Trauma & 11 & 3.6 \\
\hline & COPD & 29 & 9.5 \\
\hline & DM & 30 & 9.9 \\
\hline & Malignancy & 33 & 10.9 \\
\hline & Pulmonary edema & 17 & 5.6 \\
\hline & GIS hemorrhage & 14 & 4.6 \\
\hline & CAD & 26 & 8.6 \\
\hline \multicolumn{2}{|l|}{ Age } & \multicolumn{2}{|c|}{ Mean \pm Std.Dev.: $67.727 \pm \mid 5.954$} \\
\hline
\end{tabular}

CVA: Cerebrovascular Accident; DM: Diabetes Mellitus; CAD: Coroner Artery Disease; CHF: Congestive Heart Failure; COPD: Chronic Obstructive Pulmonary Disease

Citation: Canakci E, Karatas A, Kaya Y. Can blood groups, neutrophil-lymphocyte ratio and mean platelet volume predict mortality in critically ill patients developing acute kidney injury? J Anesth Crit Care Open Access. 20 I7;8(I): I-6. DOI: I0.15406/jaccoa.20 I7.08.00290
(CRP) level, white blood cell (WBC) count, neutrophil, lymphocyte and platelet values, mean platelet volume (MPV) and neutrophil lymphocyte ratio (NLR) were recorded. NLR values were categorized into three groups according to the percentage frequency among cases. Values between 0-40 were grouped as NLR 1, values between 41-60 were grouped as NLR 2, and values above 60 were grouped as NLR 3. Additionally blood groups ( $\mathrm{A}, \mathrm{B}, \mathrm{O}, \mathrm{AB})$ and $\mathrm{Rh}$ factors $\left(\mathrm{Rh}^{+}, \mathrm{Rh}^{-}\right)$ were noted for every case.

Normality assessment was made with Kolmogorov-Smirnov test, and homogeneity check of group variances was made with Levene test. Means of two independent groups were compared with t-test, whereas comparison of the means of three or more groups were made with one-way analysis of variance. Identification of the different groups was made with Tukey multiple comparison test with $5 \%$ significance level. Results of Tukey test were expressed in letters along with the descriptive statistics results. Calculations and interpretations were made at $5 \%$ significance level. All calculations were performed using SPSS v24 (IBM Inc., Chicago, IL, USA) statistics package software.

\section{Results}

Frequency distribution of patients according to examined parameters are presented in Table 1. One-way analysis of variance was performed in order to determine whether the studied parameters showed any difference according to blood groups. The results are presented in Table 2. As it is shown in Table 2, variance analysis for BUN 1 indicates there is no statistically significant difference between the blood groups ( $>0.05)$. Similarly, there is no significant difference between the blood groups regarding other variables $(p>0.05)$. Therefore, studied parameters did not show any difference according to the blood groups. It is concluded that blood groups are not predictors for AKI development. Studies with larger sample size are required to determine the effect of blood groups on AKI development. Student $\mathrm{t}$-test was performed in order to determine whether the studied parameters showed any change according to sex, and the results are presented in Table 3 .

\section{Statistical analysis}


Table 2 Descriptive statistics and ANOVA results for the variables according to $A B O$ groups

\begin{tabular}{|c|c|c|c|c|c|c|c|c|c|}
\hline \multirow{2}{*}{ Variables } & \multicolumn{2}{|l|}{$0(n=56)$} & \multicolumn{2}{|c|}{$A(n=\mid 87)$} & \multicolumn{2}{|c|}{$B(n=36)$} & \multicolumn{2}{|c|}{$A B(n=2 I)$} & \multirow{2}{*}{ P-Value } \\
\hline & Mean & SD & Mean & SD & Mean & SD & Mean & SD & \\
\hline$\overline{B U N I}$ & 42,786 & 23,593 & 43,255 & 25,753 & 44,000 & 28,354 & 45,095 & 29,665 & $0,986 \mathrm{NS}$ \\
\hline BUN 2 & 35,460 & 16,559 & 32,683 & 16,536 & 36,611 & 16,517 & 35,343 & $|7,37|$ & $0,456 \mathrm{NS}$ \\
\hline Crel & 1,970 & I,482 & 4,681 & 35,507 & $\mathrm{I}, 804$ & I,672 & 5,971 & 15,043 & 0,872 NS \\
\hline Cre2 & I,743 & 1,450 & 2,232 & 6,349 & 1,647 & 1,206 & 2,091 & I,548 & 0,882 NS \\
\hline GFRI & 56,804 & $43,4 \mid 4$ & 56,182 & 41,350 & 62,767 & 46,365 & 56,000 & 49,159 & $0,866 \mathrm{NS}$ \\
\hline GFR2 & 52,321 & 39,154 & 58,286 & 39,289 & 59,972 & 39,874 & 46,238 & 39,374 & $0,437 \mathrm{NS}$ \\
\hline CRP & 7,518 & 5,916 & 7,397 & 5,990 & 8,161 & 6,437 & 7,760 & 6,715 & $0,917 \mathrm{NS}$ \\
\hline WBC & 13,597 & 13,059 & 15,294 & 14,072 & 11,418 & 7,600 & $|3,05|$ & 9,316 & $0,361 \mathrm{NS}$ \\
\hline Neutrofil & 30,199 & 31,079 & 32,891 & 32,065 & 31,542 & 31,700 & 37,919 & 35,507 & $0,815 \mathrm{NS}$ \\
\hline Lymphosit & 6,048 & 7,058 & 8,672 & 29,429 & 7,894 & 9,088 & 5,269 & 5,929 & 0,85 I NS \\
\hline Platelet & $2,69,923$ & $|, 38,96|$ & $2,90,344$ & $\mathrm{I}, 45,426$ & $2,69,469$ & $\mathrm{I}, 45,743$ & $2,73,048$ & $1,34,710$ & $0,7 \mid 2 \mathrm{NS}$ \\
\hline MPV & $8,|3|$ & 1,924 & 12,197 & 49,404 & 8,252 & 2,813 & 9,034 & 2,723 & 0,875 NS \\
\hline NLR & 28,916 & 30,184 & 30,966 & 59,948 & 25,609 & 29,886 & 29,443 & 34,622 & 0,949 NS \\
\hline
\end{tabular}

SD: Standard deviation; ${ }_{N S}$ : Statistically not significant $(p>0,05)$.

Table 3 Descriptive statistics and Student's t-test results for the variables according to Sex

\begin{tabular}{|c|c|c|c|c|c|c|}
\hline \multirow{2}{*}{ Variables } & \multicolumn{2}{|c|}{ Male $(n=\mid 44)$} & \multicolumn{2}{|c|}{ Female $(n=156)$} & \multirow{2}{*}{ t-Value } & \multirow{2}{*}{ P-Value } \\
\hline & Mean & SD & Mean & SD & & \\
\hline BUN I & 41,063 & 25,221 & 45,530 & 26,318 & $-1,499$ & $0,135 \mathrm{NS}$ \\
\hline BUN 2 & 31,752 & 15,887 & 35,805 & 17,025 & $-2,127$ & $0,034 *$ \\
\hline Cre I & 2,227 & 5,926 & 5,483 & 38,854 & $-0,995$ & $0,321 \mathrm{NS}$ \\
\hline Cre 2 & 2,107 & 6,851 & 2,018 & $2,56 I$ & 0,153 & 0,879 NS \\
\hline GFR I & 62,097 & 42,498 & 52,440 & 42,606 & $\mathrm{I}, 964$ & 0,05 I NS \\
\hline GFR 2 & 64,050 & 41,178 & 49,591 & 36,295 & 3,232 & $0,001 * *$ \\
\hline CRP & 7,086 & 6,211 & 7,953 & 5,900 & $-1,239$ & $0,216 \mathrm{NS}$ \\
\hline WBC & 12,949 & 12,966 & 15,653 & 12,932 & $-1,807$ & $0,072 \mathrm{NS}$ \\
\hline Neutrofil & 30,700 & 31,495 & 34,313 & 32,419 & $-0,978$ & $0,329 \mathrm{NS}$ \\
\hline Lymphosit & 8,989 & 22,213 & 6,801 & 25,010 & 0,799 & $0,425 \mathrm{NS}$ \\
\hline Platelet & $2,78,489$ & $1,39,449$ & $2,86,811$ & $|, 46,89|$ & $-0,502$ & $0,616 \mathrm{NS}$ \\
\hline MPV & 13,154 & 56,217 & 8,518 & 3,702 & 1,028 & $0,305 \mathrm{NS}$ \\
\hline NLR & 23,110 & 28,944 & 36,040 & 64,378 & $-2,212$ & $0,028 *$ \\
\hline
\end{tabular}

SD: Standard deviation; Ns: Statistically not significant ( $>0,05)$; *: Statistically significant $(p<0,05)$; **: Statistically significant $(p<0,01)$.

As it is shown in Table 3, mean BUN 1 level was slightly higher in females than in males; however, the difference was not statistically significant according to t-test $(\mathrm{p}>0.05)$. Mean BUN 2 level was significantly higher in females compared to males $(\mathrm{p}<0.05)$. Similarly, NLR was significantly higher in females compared to males $(p<0.05)$. Similarly, other variables did not show significant difference between the blood groups $(\mathrm{p}>0.05)$. Therefore, blood group is not important factor for the variables examined in this study. Mean GFR 2 level was statistically higher in males compared to females $(p<0.01)$. Other variables did not show statistically significant difference between the sexes ( $p>0.05)$. This can be explained by the relationship of creatinine level with the muscle mass in the body, and additionally because there was not so much difference between the initial and last creatinine measurement values as it is present in BUN level. In addition, although the GFR shows greater change during the initial stages of the renal function loss, the expected change in creatinine levels is smaller.

In order to determine whether other parameters showed any change according to presence of AKI, Student t-test was applied, and the results are presented in Table 4. As shown in Table 4, mean levels of Cre1, Cre2, lymphocyte and MPV did not change depending on the presence of AKI $(\mathrm{p}>0.05)$. Student t-test results showed that mean levels of BUN1, BUN2, CRP, WBC, Neutrophil and NLR were significantly higher among patients with AKI, compared to the non-AKI group $(\mathrm{p}<0.001 / \mathrm{p}<0.05)$. In contrast, mean levels of GFR1, GFR2 and platelet were significantly higher in the non-AKI group compared to the AKI group $(\mathrm{p}<0.001)$. These statistical results are in perfect agreement with our clinical observations. We observed increased mortality risk in patiens with AKI. This can be explained by the relationship between creatinine level and body muscle mass, and because there was not so much difference between initial and last creatinine measurement values as compared to BUN levels. In addition, although the GFR shows greater change during the initial stages of the renal function loss, the expected change in creatinine levels is smaller. Furthermore, creatinine levels may be misleading in patients with sepsis. In order to determine whether the studied parameters showed any change according to discharge situation, Student t-test was applied, and the results are presented in Table 5. As shown in Table 5, mean levels of Cre1, Cre2, CRP, neutrophil, lymphocyte and MPV did not show change depending on the discharge states of patients $(p>0.05)$. Student $t$-test results indicate that mean levels of BUN 1, BUN2, WBC and NLR were significantly higher among the deceased patients compared to the discharged patients $(p<0.001 / p<0.01)$. This statistical result is in perfect agreement with our clinical observations. Indeed, elevated levels of BUN 1, BUN 2, WBC and NLR values are commonly observed in patients who end up with mortality. Because, bacteremia can develop in many critically ill patients; and renal perfusion can deteriorate due to hypotension, BUN levels can increase. Furthermore, bacteremia causes neutrophilia and increased white blood cell count. This supports our hypothesis which states that increased NLR can predict mortality. By contrast, mean levels of GFR1, GFR2 and platelet were significantly higher in discharged patients compared to the deceased patients $(p<0.001)$. Higher mean levels of GFR 1 and GFR 2 in discharged patients, and the statistically significant difference also supports our hypothesis that states NLR is a predictor of AKI. Platelet counts were higher in discharged patients, and lower in deceased patients, and the difference was statistically significant. This may be because thrombocytopenia is more frequent in critically ill patients, and especially in mortal cases. Thrombocytopenia with accompanying sepsis and thrombocytopenia 
due to polypharmacy, or thrombocytopenia due to heparin is frequently encountered in critically ill patients. This may be the reason why mean platelet count was significantly lower in deceased patients, and why mean platelet count was higher among discharged patients since the aforementioned clinical situations are rare in discharged patients.

In order to determine whether the studied parameters showed change according to NLR groups, one-way analysis of variance was applied, and results are presented in Table 6. NLR values were categorized. Values between 0-40 were grouped as NLR1, values between 41-60 were grouped as NLR2, and values above 60 were grouped as NLR3. As it is seen from Table 6, results of variance analysis indicate that the difference between NLR groups were statistically significant for lymphocyte and MPV variables ( $>0.05$ ). Other variables did not show significant difference between the NLR groups $(\mathrm{p}<0.01 / \mathrm{p}<0.001)$. In order to determine the different groups, Tukey test was applied, and the results are expressed with letters along with the means. The fact that the studied parameters showed statistically significant difference between the NLR groups proves that NLR is a predictor of AKI and mortality in critically ill patients. The fact that GFR, BUN and Cre results became significant as NLR increased emphasizes the predictive strength of NLR in AKI patients. Mortality rate is increased as the NLR is increased. Our results prove that MPV is not a predictor of mortality in critically ill patients.

Table 4 Descriptive statistics and Student's t-test results for the variables according to AKI Groups

\begin{tabular}{|c|c|c|c|c|c|c|}
\hline \multirow{2}{*}{ Variables } & \multicolumn{2}{|c|}{ AKI $(n=\mid 42)$} & \multicolumn{2}{|c|}{ Non AKI $(n=\mid 58)$} & \multirow{2}{*}{ t-Value } & \multirow{2}{*}{ P-Value } \\
\hline & Mean & SD & Mean & SD & & \\
\hline$\overline{B U N}$ I & 59,901 & 26,899 & 28,542 & 12,426 & 13,175 & $0,000 * * *$ \\
\hline BUN 2 & 40,878 & 18,250 & 27,551 & 11,838 & 7,576 & $0,000 * * *$ \\
\hline Crel & 6,520 & 40,655 & 1,583 & 5,612 & 1,511 & $0,132 \mathrm{NS}$ \\
\hline Cre2 & 2,331 & 1,759 & 1,817 & 6,806 & 0,874 & 0,383 NS \\
\hline GFR I & 30,645 & 29,579 & 80,829 & 38,681 & $-|2,5| 6$ & $0,000 * * *$ \\
\hline GFR 2 & 35,720 & 28,385 & 75,236 & 38,434 & $-10,036$ & $0,000 * * *$ \\
\hline CRP & 8,303 & 5,462 & 6,848 & 6,485 & 2,090 & $0,038^{*}$ \\
\hline WBC & 17,234 & 14,640 & 11,768 & 10,724 & 3,713 & $0,000 * * *$ \\
\hline Neutrophil & 36,565 & 33,702 & 28,996 & 29,999 & 2,058 & $0,040 *$ \\
\hline Lymphosit & 5,399 & 21,558 & 10,055 & 25,329 & $-1,705$ & 0,089 \\
\hline Platelet & $2,42,249$ & I,26,987 & $3,19,276$ & $\mathrm{I}, 47,44 \mathrm{I}$ & $-4,822$ & $0,000 * * *$ \\
\hline MPV & 8,344 & 2,418 & 12,899 & 53,733 & $-1,009$ & $0,3 \mid 4$ NS \\
\hline NLR & 43,366 & 45,632 & 17,672 & 52,439 & 4,504 & $0,000 * * *$ \\
\hline
\end{tabular}

$S D$ : Standard deviation; Ns: Statistically not significant ( $>>0,05)$; *: Statistically significant $(p<0,05)$; ***: Statistically significant $(p<0,00 \mathrm{I})$.

Table 5 Descriptive statistics and Student's t-test results for variables according to Discharge situation

\begin{tabular}{|c|c|c|c|c|c|c|}
\hline \multirow{2}{*}{ Variables } & \multicolumn{2}{|c|}{ Ex $(n=\mid 46)$} & \multicolumn{2}{|c|}{ Discharge $(n=\mid 54)$} & \multirow{2}{*}{ t-Value } & \multirow{2}{*}{ P-Value } \\
\hline & Mean & SD & Mean & SD & & \\
\hline BUN I & 58,932 & 27,227 & 28,647 & $12,4 \mid 7$ & $|2,50|$ & $0,000 * * *$ \\
\hline BUN 2 & 40,909 & 18,130 & 27,176 & $|I, 56|$ & 7,864 & $0,000 * * *$ \\
\hline Crel & 6,381 & 40,098 & $\mathrm{I}, 587$ & 5,685 & $\mathrm{I}, 468$ & $0,143 \mathrm{NS}$ \\
\hline Cre2 & 2,346 & I,759 & $|, 79|$ & 6,887 & 0,945 & $0,345 \mathrm{NS}$ \\
\hline GFRI & $31,64 \mid$ & 30,498 & 81,188 & 38,583 & $-12,296$ & $0,000 * * *$ \\
\hline GFR2 & 35,460 & 28,701 & 76,508 & 37,624 & $-10,583$ & $0,000 * * *$ \\
\hline CRP & 8,065 & 5,499 & 7,036 & 6,520 & 1,474 & 0,142 NS \\
\hline WBC & 16,995 & 14,510 & 11,852 & 10,847 & 3,489 & $0,001 * *$ \\
\hline Neutrofil & 36,261 & 33,723 & 29,088 & 29,921 & 1,951 & $0,052 \mathrm{NS}$ \\
\hline Lenfosit & 5,446 & 21,286 & 10,131 & 25,633 & $-1,717$ & $0,087 \mathrm{NS}$ \\
\hline Platelet & $2,42,269$ & $\mathrm{I}, 26,500$ & $3,21,258$ & $1,47,806$ & $-4,961$ & $0,000 * * *$ \\
\hline MPV & 8,357 & 2,391 & 13,005 & 54,426 & $-|, 03|$ & $0,303 \mathrm{NS}$ \\
\hline NLR & 42,926 & 45,428 & 17,422 & 52,811 & 4,473 & $0,000 * * *$ \\
\hline
\end{tabular}

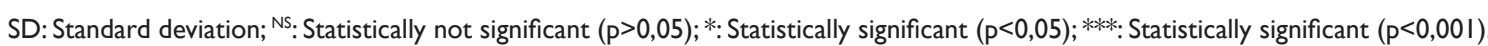

Table 6 Descriptive statistics and ANOVA results for the variables according to NLR groups

\begin{tabular}{|c|c|c|c|c|c|c|c|}
\hline \multirow[t]{2}{*}{ Variables } & \multicolumn{2}{|c|}{$\begin{array}{l}\text { NLR I }(n=223) \\
\text { Low }(0-40)\end{array}$} & \multicolumn{2}{|c|}{$\begin{array}{l}\text { NLR } 2(n=33) \\
\text { Moderate }(4 \mid-60)\end{array}$} & \multicolumn{2}{|c|}{$\begin{array}{l}\text { NLR } 3(n=44) \\
\text { High (6I above) }\end{array}$} & \multirow[t]{2}{*}{ P-Value } \\
\hline & Mean & SD & Mean & SD & Mean & SD & \\
\hline$\overline{B U N}$ I & $38,106 B$ & 23,957 & $59,909 A$ & 19,338 & $57,750 \mathrm{~A}$ & 28,988 & $0,000 * * *$ \\
\hline BUN 2 & $31,388 B$ & 15,389 & $32,955 B$ & 16,294 & $47,064 A$ & 16,786 & $0,000 * * *$ \\
\hline Crel & I,987B & 4,875 & I8,897 A & 84,046 & $2,484 \mathrm{~B}$ & I,980 & $0,000 * * *$ \\
\hline Cre2 & I,538B & 2,148 & $2,64 \mid A B$ & 2,433 & $4,275 A$ & 12,033 & $0,000 * * *$ \\
\hline GFRI & $67,422 \mathrm{~A}$ & 43,596 & $16,685 B$ & 12,292 & $34,932 \mathrm{~B}$ & 21,869 & $0,005 * *$ \\
\hline GFR2 & $64,665 \mathrm{~A}$ & 41,094 & $4 I, 642 B$ & 22,852 & $26,477 B$ & 14,373 & $0,004 * *$ \\
\hline CRP & $7,08 \mathrm{IB}$ & 6,241 & $12,242 \mathrm{~A}$ & 4,747 & $6,3 \mid 8 B$ & 4,197 & $0,000 * * *$ \\
\hline WBC & $11,359 \mathrm{C}$ & 8,008 & $27,575 \mathrm{~A}$ & 14,234 & $19,624 \mathrm{~B}$ & 21,995 & $0,000 * * *$ \\
\hline Neutrofil & $24,939 B$ & 26,770 & $54,773 \mathrm{~A}$ & 35,353 & $54,653 \mathrm{~A}$ & 36,067 & $0,000 * * *$ \\
\hline Lymphocyt & 7,956 & 21,680 & 10,552 & 43,852 & 5,293 & 5,816 & $0,625 \mathrm{NS}$ \\
\hline Platelet & $301,747 A$ & $\mathrm{I}, 39,976$ & $242,20 \mathrm{IAB}$ & $1,04,820$ & $217,336 \mathrm{~B}$ & $\mathrm{I}, 60,387$ & $0,000 * * *$ \\
\hline MPV & II,64IA & 45,193 & $8,54 \mathrm{~B} 5$ & 2,686 & $7,840 \mathrm{~B}$ & 5,536 & $0,793 \mathrm{NS}$ \\
\hline NLR & $10,247 C$ & 10,243 & $58,825 B$ & 6,811 & $107,362 \mathrm{~A}$ & 93,033 & $0,000 * * *$ \\
\hline
\end{tabular}

SD: Standard deviation; Ns: Statistically not significant $(p>0,05)$;

*: Statistically significant $(\mathrm{p}<0,05)$; $* * *$ : Statistically significant $(\mathrm{p}<0,00 \mathrm{I})$.

Means that do not share a letter are significantly different $(P<0,05)$ 


\section{Discussion}

Acute kidney injury (AKI) is a clinical syndrome in which there is often reversible reduction of GFR accompanied by accumulation of nitrogen metabolites such as urea and creatinine, fluid and electrolyte imbalances, and disorders of acid base metabolism. It is gaining increasing importance due to the high prevalence in critically ill patients and its close association with mortality. ${ }^{19}$ Many different factors may play role in the etiology of AKI. One of the leading cause of AKI observed in critically ill patients is sepsis. In one study by Palmar et al. ${ }^{20}$ the prevalence of AKI was reported as $19 \%$ in sepsis, $23 \%$ in severe sepsis, and $51 \%$ in septic shock. ${ }^{20}$ In another epidemiological study involving patients diagnosed with AKI, Wang et al. found that more than $50 \%$ of the patients had accompanying sepsis or septic shock. In our study, $60 \%$ of the patients with AKI had septic shock. ${ }^{21}$ High NLR, CRP and WBC levels in our cases suggest presence of bacteremia and sepsis in majority of our cases. According to our clinical observations, most of the cases diagnosed with AKI have accompanying bacteremia or sepsis. Our results are in agreement with the literature data. Presence of AKI significantly increases the risk of mortality in intensive care, and mortality rate is increased up to $90 \%$ in case renal replacement therapy is required. ${ }^{22}$ In one study including 211 patients with sepsis, Peng et al. found significantly increased 28-day mortality rate in the AKI group. ${ }^{23}$ In the present study, the comparison of patients with and without AKI showed higher mortality rate in patients with AKI. This result is consistent with the literature data. Studies that examined AKI development during ICU stay have found that RIFLE and AKIN had significant effects on in-hospital survival; and no difference was found between the two classifications with regard to their ability to predict survival, ${ }^{24}$ Park et al., ${ }^{25}$ evaluated AKI development in ICU patients based on RIFLE classification alone, and they found significant effect of RIFLE on hospital survival. ${ }^{25}$ In the present study, we also used RIFLE classification for diagnosing AKI.

Whether there is an association between blood groups and various diseases, or in other words, whether some blood groups are associated with higher risk for some diseases has been investigated by many researchers. ${ }^{26,27}$ In literature, its associations with some infectious diseases, some cancer types and especially cardiovascular diseases have been investigated. Kaya et al. found lower risk of coronary artery disease in individuals with blood group $\mathrm{O}$, whereas this risk was found to be higher with $\mathrm{A}, \mathrm{B}$ and $\mathrm{AB}$ blood groups. ${ }^{27}$ In the present study, we did not find association of blood groups with mortality or AKI. Additionally, the present study is to first study to examine the relationship of blood groups with mortality and AKI. Considering that the present study included a sample size of 300 patients and data collected over 7years, it is obvious that studies with even larger sample size, and longer study period are needed to demonstrate any possible association with blood groups.

In our review of literature, we encountered many studies examining whether NLR is a predictor of various diseases. A great number of these studies are related with the association with coronary artery disease. One study from Michigan University, USA, evaluated NLR values measured at the time of admission and all-cause mortality at 6th month in 2833 patients diagnosed with acute coronary syndrome (ACS) (564 ACS with ST-segment elevation, 2269 ACS with unstable angina pectoris/ non-ST-segment elevation); and similar to our results, NLR was found as an effective predictor of mortality. That study had the largest sample size in related literature. ${ }^{28}$ One study that evaluated NLR values measured at the time of presentation and in-hospital and all-cause 30th day mortality found that NLR was an effective parameter in only Type 2 diabetes. ${ }^{29}$ Studies investigating the association with mortality evaluated in-hospital, post-discharge, 30th day and 6th month mortality rates. ${ }^{28,29}$ The study by Azab et al. from Staten University (Staten Island, New York, USA) had the longest follow-up period. ${ }^{30}$ This study evaluated NLR values at presentation and mean NLR values (average NLR value calculated from 3 different differential white blood cell count on different days) in 619 ACS patients with unstable angina pectoris/ non-ST segment elevation, and 4th year mortality rates from USA Social Security Mortality Index records. ${ }^{28,29}$ Our study had a retrospective design, and a comparison regarding follow-up time cannot be made. However, our results in terms of the association between NLR and mortality are in agreement with the literature data.

There are few studies that examined the relationship between AKI and NLR. Considering this fact, our study is among the first few studies related with AKI and NLR. Besides this good situation, but unfortunately there are some limitations of our study. It is primarily a retrospective file review, so we can not predict the degree of correlation with clinical outcomes. We could grade kidney injury according to the RIFLE or KDIGO guide. We still believe that the results are in accordance with the literature. In one study involving patients diagnosed with AKI and severe sepsis, Y1lmaz et al., ${ }^{31}$ reported that NLR is a predictor with higher sensitivity and specificity compared to CRP or WBC. ${ }^{31}$ Their study was a 3-year retrospective study including 118 critically ill patients, whereas our study is 7-year retrospective study including 300 patients. In comparison to the study by Y1lmaz et al., larger sample size and longer study period bestow more strength to the present study. Analyzed laboratory parameters, demographic variables and accompanying comorbidities show great similarity between the studies. On the other hand, one superior side of the study by Yilmaz et al. is that they included APACHE II and SOFA scores of the patients in the analysis. Since the ICU in our hospital is categorized as grade II ICU, we could not obtain APACHE II and SOFA scores of all our patients, therefore these scores were not included in analysis. Because Yilmaz et al. used these intensive care scoring systems, their study is superior in this regard. ${ }^{31}$ In one study involving hemodialysis patients, Neuen et al., ${ }^{31}$ stated that NLR was a simple and inexpensive predictor of mortality. They followed up 170 hemodialysis patients for 3 weeks, and they found NLR could predict all-cause mortality, and particularly mortality due to cardiovascular reasons. Additionally, they found positive correlation between increased CRP and increased NLR levels. ${ }^{32}$ The study results of Neuen et al., ${ }^{32}$ are in complete agreement with our results. In our study, we also found significant results for CRP, platelet, WBC and neutrophil values in addition to NLR when cases with AKI were compared to the non-AKI group. ${ }^{32}$ In their letter to editor, Balta et al. criticized some aspects of the study by Y1lmaz et al. Balta et al. stated that NLR could not be a predictor in cases with AKI, because NLR can be affected by many factors including the methods used for phlebotomy, and NLR could be lower in patients receiving statin treatment and antihypertensive treatment (valsartan, nebivolol, amlodipin), and they stated that the results reported by Y1lmaz et al., ${ }^{31}$ were not reliable, and that these results should be supported with other serum inflammation markers. ${ }^{33}$ In our opinion, the critical reviews put forward by Balta et al. are not justifiable. Y1lmaz et al. could achieve a homogenous sample, and included critically ill patients with concurrent severe sepsis and AKI. These cases with severe sepsis do not receive antihypertensive medications, instead, they are hypotensive patients that require positive inotropic support. Moreover, the authors even detected the origin of infection in cases diagnosed with severe sepsis. Additionally, Yilmaz et al., ${ }^{31}$ categorized NLR values, and NLR values were found in a close range. For this reason, we think the criticisms by Balta et al. have no ground. Our study is the third study to evaluate the association between NLR 
and AKI, coming after the studies by Y1lmaz et al., ${ }^{31}$ and Neuen et al., ${ }^{32}$ and our results are in complete agreement with their results.

Güldiken et al., ${ }^{34}$ compared MPV values and other peripheral blood cell counts in cases with acute ischemic stroke, and they reported that although MPV did not show significant change in acute ischemic stroke, increased leukocyte and neutrophil counts were good indicators of major vessel disease subtype and stroke severity. In our study, we did not find association between MPV values and AKI or mortality, ${ }^{34}$ In one study, Karagöz et al., ${ }^{35}$ categorized critically ill patients in two groups as deceased and discharged patients, and they examined the associations of hemogram parameters and especially MPV with mortality. They found strong association between increased MPV values and mortality. ${ }^{35}$ Their results are not in agreement with our results. In our study, when we compared discharged and deceased cases, we found statistically significant results for platelet, NLR, GFR and BUN levels, whereas we did not find significant association between MPV and mortality. The study by Karagöz et al., ${ }^{34}$ is a retrospective study including critically ill patients, and they did not conduct their study on a specific disease group. The reason why we had different results may be related to the fact that we included cases with a specific disease. In one study, Farah and Samra ${ }^{36}$ compared NLR and MPV values between cases diagnosed with ischemic stroke and 30 healthy volunteers, and they found higher NLR values in cases with ischemic stroke compared to healthy volunteers, whereas they did not find difference in MPV values. Farah and Samra ${ }^{36}$ proposed that NLR was a good predictor for stroke and prognosis of stroke, while MPV was not. ${ }^{36}$ Their results are in complete agreement with our results. We also found that while NLR was a good predictor for mortality, MPV did not have a predicting value. In their study, Liu et al. investigated the association between MPV and mortality in cases with acute myocardial infarction. In that retrospective study including 567 cases, they concluded that MPV was a predictor for mortality. Their results are in contrast with our results. This may be because they had a larger sample size and included a different patient group. ${ }^{37}$ In another study, Yilmaz $\mathrm{G}$ et al. ${ }^{38}$ compared NLR and MPV values and their relationship with proteinuria level between cases with chronic renal failure (CRF) and healthy volunteers. While there was statistically strong correlation between NLR level and proteinuria level in cases with CRF, there was no correlation between MPV and proteinuria levels. ${ }^{38}$ These results are in agreement with our results. Our results are consistent with literature data. There are few studies examining the association between MPV and intensive care mortality, therefore our study is among the first studies on this subject.

\section{Conclusion}

In conclusion, considering the literature knowledge and our study results, it can be stated that MPV and blood groups are not predictors for AKI or mortality in critically ill patients, whereas NLR appears to be a strong predictor of AKI and mortality in critically ill patients. It should be emphasized that the NLR ratio should be calculated for each hospitalized ICU patient and if the ratio is high, it is likely that kidney injury may develop and measures should be taken accordingly. We believe that NLR ratio should be included in follow-up and treatment of intensive care patients.

\section{Acknowledgements}

We wish to thank Assist. Prof. Dr. YelizKasko Arıcı (Ph.D) for helping us with the statistical analyses of the present study.

\section{Conflicts of interest}

Author declares there are no conflicts of interest.

\section{References}

1. Chertow GM, Burdick E, Honour M, et al. Acute Kidney Injury, Mortality, Length of Stay, and Costs in Hospitalized Patients. J Am Soc Nephrol. 2005;(11):3365-70.

2. Hoste EA, Clermont G, Kersten A, et al. RIFLE criteria for acute kidney injury are associated with hospital mortality in critically ill patients: a cohort analysis. Crit Care. 2006;10(3):R73.

3. Lameire N. The definitions and staging systems of acute kidney injury and their limitations in practice.Arab J Nephrol Transplant. 2006; $6(3): 145-152$

4. Hoste EA, KellumJA. Incidence, classification, and outcomes of acute kidney injury.ContribNephro . 2007;156:32-38.

5. Kellum JA, Levin N, Bouman C, et al. Developing a consensus, classification system for acute renal failure. Crit Care. 2002;8(6):509-514

6. Bellomo R, Ronco C, Kellum JA, et al. and the ADQI workgroup Acute renal failure - definition, outcome measures, animal models, fluid therapy and information technology needs: the Second International Consensus Conference of the Acute Dialysis Quality Initiative (ADQI) Group. Crit Care. 2004;8(4):R204-212.

7. Metnitz PG, Krenn CG, Steltzer H, et al. Effect of acute renal failure requiring renal replacement therapy on outcome in critically ill patients. Crit Care Med. 2005;30(9):2051-2081.

8. Uchino S, Kellum JA, Bellomo $\mathrm{R}$, et al. Acute renal failure in critically ill patients: a multinational, multicenter study. JAMA. 2005; 294(7):813-818

9. Zahorec R. Ratio of neutrophil to lymphocyte counts - rapid and simple parameter of systemic inflammation and stress in critically ill.Bratis Lek Listy. 2001;102(1):5-14.

10. Perez DR, Baser RE, Cavnar MJ, et al. Blood Neutrophil-to-Lymphocyte Ratio is Prognostic in Gastrointestinal Stromal Tumor. Ann Surg Oncol. 2013;20(2):593-599.

11. ekimsoy Z, Payzin B, Örnek T, et al. Mean platelet volume in Type 2 diabetic patients.J Diabetes Complications. 2004;18(3):173-176.

12. Bancroft AJ, Abel EW, Mclaren M, et al. Mean platelet volume is a useful parameter:areproducible routine method using a modified Coulter Thrombocytometer.platletes. 2000;11(7):379-387.

13. Şenaran H, İleri M, Altınbaş A, et al. Thrombopoietin and mean platelet volume in coronary artery disease.Clin Cardiol. 2001;24(5):405-408.

14. Henning BF, Zidek W, Linder B, et al. Mean platelet volume andcoronary heart disease in hemodialysis patients. Kidney Blood Press Res. 2002;25(2):103-108.

15. Papanas N, Symeonidis G, Maltezos E, et al. Mean platelet volume in patients with type 2 diabetes mellitusPlatelets. 2004;15(8):475-478.

16. Coban E, Yazicioğlu G, BerkantAvci A, et al. The mean platelet volume in patients with essential and white coat hypertension.Platelets. $2005 ; 16(7): 435-438$

17. Sari I, Ozer O, Davutoglu V, et al. ABO blood group distribution and major cardiovascular risk factors in patients with acute myocardial infarction.Blood Coagul Fibrinolysis. 2008;19(3):231-234.

18. Wu O, Bayoumi $\mathrm{N}$, Vickers $\mathrm{MA}$, et al. $\mathrm{ABO}(\mathrm{H})$ blood groups and vascular disease: a systematic review and meta-analysis.J Thromb Haemost. 2008;6(1):62-69.

19. Wang H, Muntner P, Chertow G, et al. Acute Kidney Injury and Mortality in hospitalized Patients.Am J Nephrol. 2022;35(4):349-355.

20. Palmar A, Langenberg C, Wan L, , et al. Epidemiology of septic acute kidney injury. Curr Drug Targets. 2009;10(12):1169-1178. 
21. Wang X, Jiang L, Wen Y, et al. Risk Factors for Mortality in Patients with Septic Acute Kidney Injury in Intensive Care Units in Beijing, China: A Multicenter Prospective Observational Study. Biomed Res Int. 2014;1-10.

22. Oruç A, Ersoy A, Hoyrazlı A, et al. Should Hemodialysis for Renal Replacement Treatment in Hospitalized Patients with Acute Kidney Injury be Intermittent or Continuous Turk Neph Dial Transp. 2012;21(1):72-77.

23. Peng Q, Zhang L, Ai Y, et al. Epidemiology of acute kidney injury in intensive care septic patients based on KDIGO guidelines. Chin Med J. 2014;127(10):1820-1826.

24. Lopes JA, Fernandes P, Jorge S, et al. Acute kidney injury in intensive care unit patients: a comparison between the RIFLE and the Acute Kidney Injury Network classifications. Crit Care. 2008;12(4):R1- R110.

25. Park WY, H wang EA, Jang MH, et al. The risk factors and outcome of acute kidney injury in the intensive care units. Korean J Intern Med. 2010;25(2):18118-7.

26. Juhl BR. Blood group antigens in transitional cell tumours of the urinary bladder. An immunohistochemical study. Dan Med Bull. 1994; 41(1):1-11.

27. Kaya A, Tanboğa İH, Kurt M, et al. Relation of ABO blood groups to coronary lesion complexity in patients with stable coronary artery disease. AnadoluKardiyolDerg. 2014;14(1):55-60.

28. Muhammed Suliman MA, BahnacyJuma AA, Ali Almadhani AA, et al Predictive value of neutrophil to lymphocyte ratio in outcomes of patients with acute coronary syndrome. Arch Med Res. 2010;41(8):618-622.

29. Tamhane UU, Aneja S, Montgomery D, et al. Association between admission neutrophil to lymphocyte ratio and outcomes in patients with acute coronary syndrome.Am J Cardiol. 2008;102(6):653-657.
30. Azab B, Zaher M, Weiserbs KF Torbey E, et al. Usefulness of neutrophil to lymphocyte ratio in predicting short- and long-term mortality after non-ST-elevation myocardial infarction. Am J Cardiol. 2010; 106(4): 470-476.

31. Yilmaz H, Cakmak M, Inan O, et al. Can neutrophil-lymphocyte ratio be independent risk factor for predicting acute kidney injury in patients with severe sepsis? Ren Fail. 2015;37(2):225-229.

32. Neuen BL, Leather N, Greenwood MA, et al. Neutrophil-lymphocyte ratio predicts cardiovascular and all-cause mortality in hemodialysis patients. Ren Fail. 2016;38(1):70-76.

33. Balta S, Uslu AU, Unlu $\mathrm{M}$, et al. The relation between neutrophil-lymphocyte ratio and acute kidney injury. Ren Fail . 2015;37(9):1527-1528.

34. Güldiken B, Ozkan H, Kabayel L.

35. Sayısı Yanıtı Akut İskemik İnmede Ortalama Trombosit Hacmive Periferik Kan Hücre. Trakya Univ Tip Fak Derg. 2008;25(2):130-135.

36. Karagoz I, Aktas G, Yoldas H, et al. Association Between Hemogram Parameters and Survival of Critically Ill Patients J Intensive Care Med. 2017.

37. Farah R, Samra N. Mean platelets volume and neutrophil to lymphocyte ratio as predictors of stroke. J Clin Lab Anal. 2017.

38. Liu X, Wang S, Yuan L, et al. Increased Mean Platelet Volume is Associated with Higher In-Hospital Mortality Rate in Patients with Acute Myocardial Infarction. Clin Lab. 2017;63(1):163-167.

39. Yilmaz G, Sevinc C, Ustundag S, et al. The Relationship between Mean Platelet Volume and Neutrophil/Lymphocyte Ratio with Inflammation and Proteinuria in Chronic Kidney Disease .Saudi J Kidney Dis Transpl. 2017;28(1):90-94 